\title{
A C. elegans platform for the discovery of opioid modulators
}

Wang, D. et al. Science 365, 1267-1273 (2019)

Opioids are extensively used to relieve pain, but their addictive properties can lead to dependence and abuse. Evidence shows that potent opioids such as fentanyl and morphine act on the $\mu$-opioid receptor (MOR), a G protein-coupled receptor (GPCR), but the downstream signaling pathways are not fully understood. Efforts are currently underway to better understand the endogenous MOR system and prevent the adverse effects of opioidinduced MOR activation. Using the worm Caenorhabditis elegans as a screening platform to find genes that can modulate MOR functions, a team of investigators led by Brock Grill and Kirill Martemyanov at the Scripps Research Institute identified a new orphan receptor that negatively regulates MOR signaling. These findings could be used to improve opioid therapy safety.

Knowing that opioid agonists and MOR activation affect motor activity, the investigators decided to perform a forward genetic screen on C. elegans and identify regulators of MOR signaling by using worm movements as an ultimate readout.

They generated transgenic C. elegans expressing mammalian MOR throughout the nervous system (tgMOR) and showed that exposure to fentanyl and morphine inhibited motor activity in tgMOR worms. Based on the observation that a greater sensitivity to opioids leads to faster paralysis and greater recovery, the researchers performed a mutagenesis screen and analysed the movements of the mutant worms. A total of $\sim 2,500$ tgMOR animals were mutagenized and $\sim 600,000$ progeny were analysed for their ability to regulate MOR activity after morphine and fentanyl exposure; this strategy led to the identification of $\sim 900$ mutants with abnormal opioid sensitivity, notably a tgMOR; $\operatorname{lgg} 9$ mutant with hypersensitivity to opioids.

Using whole-genome sequencing, the investigators identified the lesion responsible for the mutant phenotype: a mutation in frpr13 , a gene encoding an unstudied orphan GPCR conserved in mammals (GPR139). To investigate the role of GPR139 in mammals, they studied the responses of Gpr139-/mice to opioid exposure. Gpr $139^{-/-}$mice exhibited a greater sensitivity to the effects to morphine and less withdrawal symptoms than wild-type mice.

Overall, the results indicate that GPR139 negatively regulates responses to acute opioid exposure and potentiates withdrawal from chronic opioid use, which could make this molecule a target for the development of safer opioid therapy.

"Forward genetics-unbiased genetic discovery-has never been applied to probing an opioid receptor like this," Grill says in a press release. "The opioid epidemic is a huge problem and we don't have good solutions. This type of approach can bring a whole new array of targets and a new way of thinking about and going after an old problem."

\section{Alexandra Le Bras}

Published online: 14 October 2019 https://doi.org/10.1038/s41684-019-0426-3

\section{Poking hurts. Be nice.}
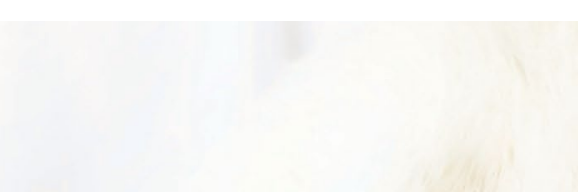